\title{
Useful variables during wake and sleep for prediction of positive airway pressure in obstructive sleep apnea titration
}

\author{
Javad Setareh', Majid Malekmohammad², Mahnaz Amini ${ }^{3}$, Batoul Khoundabi ${ }^{4}$ and Parisa Adimi Naghan ${ }^{4 *}$
}

\begin{abstract}
Background: Positive airway pressure is the main treatment modality in obstructive sleep apnea. The level of pressure needed for each patient is defined by a positive airway pressure titration study. Predicting those who need higher pressure or bi-level instead of continuous pressure is of a great clinical significance. This study was designed to evaluate whether voluntary breath-holding maneuver could predict Bilevel Positive Airway Pressure (BiPAP) or Continuous Positive Airway Pressure (CPAP) as the final optimal pressure in a split night study of patients with obstructive sleep apnea. Polysomnography (PSG) parameters specially oxygen saturation $\left(\mathrm{SaO}_{2}\right)$ during non-rapid eye movement (NREM), rapid eye movement sleep (REM), and duration of REM in diagnostic part may help determine the type of devices as soon as possible in split night study.

Methods: The present research was conducted as a cross-sectional study of adults diagnosed as obstructive sleep apnea patients undergoing positive airway pressure (PAP) titration. Demographic, anthropometric, and polysomnographic data were collected. Patients were instructed to hold their breath as long as they could after five tidal breaths in the supine position. Baseline, post breath-holding phase, and recovery $\mathrm{SaO}_{2}$ were recorded. These data were used to predict the pressure level and type of device.

Results: Seventy-eight participants ( $56.4 \%$ male) with the mean age of $55.7 \pm 13.9$ years were included in the study. Mean and SD of apnea hypopnea index (AHI) and oxygen desaturation index (ODI) were $55.9 \pm 34.4$ and $38.3 \pm 24$ per hour, respectively. Most of the participants (65.3\%) were categorized as CPAP group. CPAP and BiPAP groups were similar in terms of age, gender distribution, body mass index (BMI), neck circumference, and certain polysomnographic variables. Voluntary breath-holding maneuver showed a significant correlation between minimum $\mathrm{SaO}_{2}$ and the need for changing to BiPAP during titration. Baseline and post breath-holding $\mathrm{SaO}_{2}$ were not significantly lower in either group. NREM minimum $\mathrm{SaO}_{2}$ and $\mathrm{REM}$ duration were the statistically significant correlated variables that predicted the need for BiPAP.
\end{abstract}

Conclusion: Minimum oxygen following voluntary breath-holding maneuver along with higher BMI and larger neck circumference are predictors of the need to use BiPAP. Shorter REM duration and NREM minimum $\mathrm{SaO}_{2}$ were other predictors of higher chance of BiPAP during titration of the present OSA participants.

Keywords: CPAP, BiPAP, Obstructive sleep apnea, Oxygen saturation, Voluntary breath-holding maneuver

\footnotetext{
* Correspondence: prs_adimi@yahoo.com

${ }^{4}$ Clinical Tuberculosis and Epidemiologic Research Center, National Research

Institute of Tuberculosis and Lung Diseases (NRITLD), Shahid Beheshti

University of Medical Sciences, Tehran, Iran

${ }^{5}$ Masih Daneshvari Hospital, PO Box: 19575-154, Daar-Abad, Niavaran, Tehran

Zip Code: 19569-44413, Iran

Full list of author information is available at the end of the article
}

(c) The Author(s). 2019 Open Access This article is distributed under the terms of the Creative Commons Attribution 4.0 International License (http://creativecommons.org/licenses/by/4.0/), which permits unrestricted use, distribution, and reproduction in any medium, provided you give appropriate credit to the original author(s) and the source, provide a link to the Creative Commons license, and indicate if changes were made. The Creative Commons Public Domain Dedication waiver (http://creativecommons.org/publicdomain/zero/1.0/) applies to the data made available in this article, unless otherwise stated. 


\section{Background}

Positive Airway Pressure (PAP) is the main therapeutic method for dealing with Obstructive Sleep Apnea (OSA). Different PAP modalities have been used to help patients cope with predominant OSA usually guided by a nighttime PAP titration study. Recommended titration protocols suggest the start of titration with a minimum level of CPAP and a subsequent gradual increase to a higher level (either Bilevel-PAP or newer modalities). Accordingly, the patient would use the suitable device to optimally alleviate obstructive events (Antonescu-Turcu \& Parthasarathy, 2010; Carlucci et al., 2015; Kushida et al., 2008).In fact, PAP titration process needs expertise and time; furthermore, it is usually interrupted by multiple awakenings which can further delay the titration process or constrain a repeat titration study. Predicting the need of those groups of patients who require BIPAP can save both time and cost. Specially, in split night study, the technician is normally under time pressure to switch CPAP to BIPAP. Many formulas derived from clinical and para-clinical variables have been developed to predict the optimal PAP. Oxygen desaturation, Apnea Hypopnea Index, and neck circumference are considered to be the main predictive factors for the prediction of CPAP pressure in the body of related literature (Abdel Wahab \& Ahmed, 2017; Camacho, Riaz, Tahoori, Certal, \& Kushida, 2015; Ebben, Narizhnaya, \& Krieger, 2017; Noseda et al., 2009).

In addition to usual clinical parameters such as BMI, neck circumference, and results of survey research used in most of PAP prediction tools, researchers have some interesting clinical maneuvers at their disposal including the five-minute wake snore test to help predict PAP pressure (Camacho et al., 2016).

To the best of our knowledge, there has been no study so far investigating the role of voluntary breath-holding maneuver in the prediction process of PAP pressure. Thus, the present study was designed to see whether this maneuver along with minimum oxygen saturation during REM and NREM sleep could be helpful in deciding between CPAP and BiPAP need.

\section{Methods}

\section{Study design}

The present research was cross-sectional and was conducted from December 2016 up to December 2017 which originally included a pool of 510 patients; after exclusion of those patients who were either suffering from other conditions or could not tolerate using the devices, 78 patients with split night study were enrolled.

\section{Sample size:}

According to a pilot study with 15 patients, sample size was calculated by following formula:

$$
\begin{aligned}
n & =\frac{\left(Z_{\alpha}+Z_{\beta}\right)^{2} \times(S)^{2}}{(E)^{2}} \\
& =\frac{(1.96+0.84)^{2} \times(1.6)^{2}}{(0.5)^{2}} \approx 80
\end{aligned}
$$

In this formula $\alpha, \beta, \mathrm{S}, \mathrm{E}, Z_{\alpha}, Z_{\beta}$ are Error type I, Error type II, standard deviation of REM duration, desired margin of error, the standard normal deviate for $\alpha$ and the standard normal deviate for $\beta$ respectively. Error type I and power are 0.05 and 0.80 (Chow, Wang, \& Shao, 2003).

It involved adult OSA participants scheduled for a PAP titration study (split night study) in two sleep laboratories in Tehran, Iran.

Participants who were excluded from the research project included those who had one of the following features i.e. those unable to abide by the instructions, incapable of holding breath, having unacceptable levels of titration according to Kushida guideline (Kushida et al., 2008), having a wake $\mathrm{PaCo}_{2} \geq 55$ and $\mathrm{SaO}_{2} \leq 80 \%$, and lastly, those suffering from decompensated cardiopulmonary disorders and/or any acute medical condition (such as pain) preventing apnea maneuver or sleep study. This research was approved by the Ethics Committee of Shahid Beheshti University of Medical Sciences.

\section{Data collection}

Demographic variables (age, gender), anthropometric data (height, weight, neck circumference and body mass index (BMI) and the history of chronic medical disorders diabetes mellitus (DM), systemic hypertension (HTN), ischemic heart disease (IHD) were collected through patient examinations recorded in their medical files.

\section{Voluntary breath-holding maneuver}

Prior to the sleep study, the participants were asked to lie down in a comfortable supine position with a pulse oximeter (Massimo, USA) probe on their non-dominant index finger. The baseline steady state $\mathrm{SaO}_{2}$ was measured after taking 5 tidal breaths. Then, the participants were instructed to hold their breath as long as they could. Minimum $\mathrm{SaO}_{2}$, breath-holding $\mathrm{SaO}_{2}$, and recovery $\mathrm{SaO}_{2}$ along with the duration of breath-holding and time taken prior to $\mathrm{SaO}_{2}$ fall and recovery (in seconds) were recorded by a stopwatch.

\section{PSG study}

The night-time polysomnography (PSG level I) and the titration study (Philips Respironics Software) and (BiPAP, Respironics, USA) were scored manually according to 2016 American Academy of Sleep Medicine (AASM) criteria. Total recording time (TRT), total sleep time (TST), AHI, ODI, NREM and REM duration and minimum oxygen saturation, duration of $\mathrm{SaO}_{2}$ less than $90 \%$ $(\mathrm{T}<90 \%)$, duration of sleep in each body position, and 
the pressure level required to alleviate respiratory events were extracted from the PSG results. Participants were assigned to either the CPAP or BiPAP groups according to their optimal PAP modality. If the patient is not controlled with pressure $15 \mathrm{~cm} \mathrm{H}_{2} \mathrm{O}$, then CPAP would be changed to BIPAP.

\section{Statistical analysis}

A T-test analysis was run to assess the effect of demographics and other variables on the PAP group. A similar analysis was done to compare two groups in a non-normal situation by Mann-Whitney U Test. Meanwhile, Chi-squared test was run in contingency tables for categorical items. Besides, in order to assess the effect of variables in the allocation of participants to BiPAP group, two statistical methods were used: logistic regression and decision tree analysis. Formal measures of classification performance and model evaluation criteria including sensitivity, specificity and positive and negative likelihood ratios were taken into account. The area under the curve (AUC) of a receiver operating characteristic (ROC) curve is a way to reduce the ROC performance to a single value representing the expected performance. The data were analyzed by IBM SPSS-22.0. Probability Value of 0.05 was set to be statistically significant in two-tailed tests.

\section{Results}

A total number of 78 patients $(56.4 \%$ men) with the mean age of $55.7 \pm 13.9$ were enrolled from December 2016 to December 2017. Mean \pm SD of AHI and ODI were respectively $55.9 \pm 34.4$ and $38.3 \pm 24$ per hour. Figure 1 represents the flow chart of included and excluded participants; 51 (65.3\%) were controlled with CPAP and the remaining 27(34.7\%) needed BiPAP for avoiding respiratory events. Demographic, anthropometric, polysomnographic, and titration data of each group are represented in Table 1.

Mean and SD of CPAP pressure were $11.8 \pm 2.9$ mmHg.In BiPAP group IPAP and EPAP are $21.4 \pm 3.3$ and $15 \pm 2 \mathrm{mmHg}$ respectively. The data on voluntary breath-holding maneuver (durations and $\mathrm{SaO}_{2}$ levels) were represented in detail for each group (Table 2). Both groups were similar in terms of the PSG results $(p>0.05)$.

a) Logistic Regression: BiPAP was assumed as the reference group. Polysomnographic and breathholding variables were evaluated for their changing impact on BiPAP. Multivariate Logistic Regression Analysis (Table 3) showed statistically significant odds for BMI, neck circumference, REM Duration, Minimums NREM SaO2 and Average $\mathrm{SaO} 2$ changed from CPAP to $\operatorname{BiPAP}(1.75,1.9,1.45,1.59$, and 1.8, respectively).

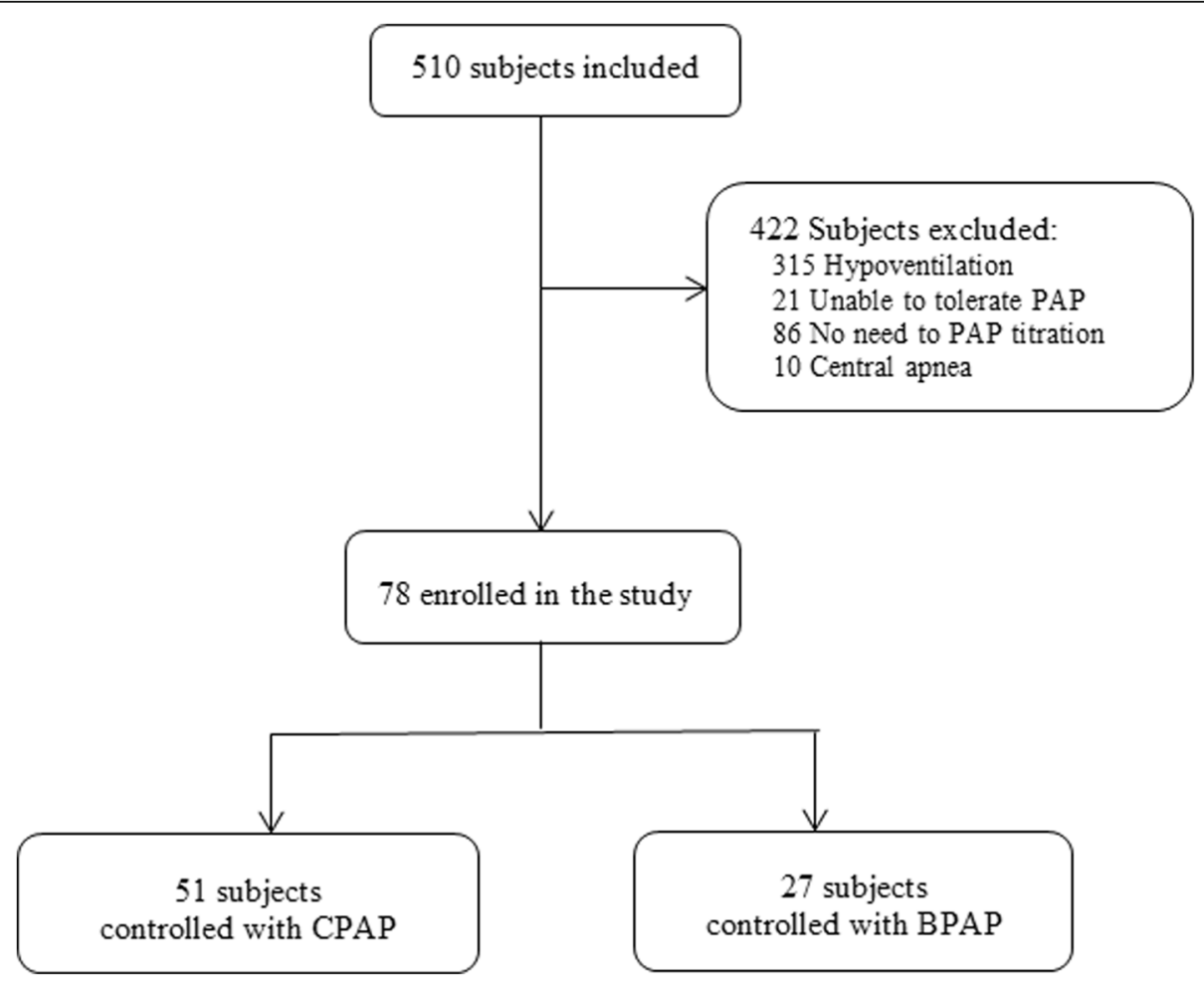

Fig. 1 flow chart showing included and excluded participants with finally 78 participants categorized as CPAP and BiPAP groups 
Table 1 Demographic, anthropometric, polysomnographic and titration data in CPAP and BiPAP groups

\begin{tabular}{llll}
\hline PAP group & $\begin{array}{l}\text { CPAP } \\
n=51\end{array}$ & $\begin{array}{l}\text { BiPAP } \\
n=27\end{array}$ & $\begin{array}{l}p \text { - } \\
\text { value }\end{array}$ \\
\hline Aariables & $53.4 \pm 13.7$ & $59.6 \pm 13.7$ & 0.057 \\
Male number (\%) & $30(59.6 \%)$ & $14(53.6 \%)$ & 0.602 \\
DM(\%) & $24(47.0 \%)$ & $9(33.3 \%)$ & 0.382 \\
HTN(\%) & $15(29.4 \%)$ & $5(18.5 \%)$ & 0.344 \\
CAD(\%) & $16(31.4 \%)$ & $9(33.3 \%)$ & 0.950 \\
Neck circumference (cm) & $41.2 \pm 3.7$ & $41.9 \pm 4.7$ & 0.427 \\
BMI (kg/m ${ }^{2} \pm$ SD) & $31.3 \pm 6.0$ & $33.0 \pm 4.8$ & 0.216 \\
AHI category (\%) & & & 0.543 \\
$\quad$ 5-14.9 & $2(3.9 \%)$ & $2(7.4 \%)$ & \\
$\quad 15-29.9$ & $11(21.6 \%)$ & $3(11.1 \%)$ & \\
$\quad \geq 30$ & $37(74.5 \%)$ & $21(81.5 \%)$ & \\
ODI & $35.6 \pm 23.0$ & $45.8 \pm 26.0$ & 0.193 \\
T<90\% ${ }^{\text {a }}$ & $11.0 \pm 18.1$ & $13.0 \pm 17.4$ & 0.964 \\
REM Cycle Number & $1.6 \pm 2.1$ & $0.7 \pm 1.0$ & 0.249 \\
Supine position & $10(19.6 \%)$ & $8(28.6 \%)$ & 0.340 \\
\hline Con & & &
\end{tabular}

Continues variable are indicated by Mean \pm SD and categorical variables are indicated

${ }^{\text {a }}$ Time of sleep with $\mathrm{SaO} 2<90 \%$

The Logistic Equation to calculate the probability of BiPAP selection as therapy device $(\hat{p})$,is suggested in the below. In this equation $x_{1}$ to $x_{5}$ represent significant explanatory variables in logistic analysis of BMI, Neck, REM duration, Minimum NREM $\mathrm{SaO} 2$ and average $\mathrm{SaO} 2$ :

$p=\frac{\exp \left(\text { intercept }+0.146 * x_{1}+0.667 * x_{2}+0.375 * x_{3}+0.465 * x_{4}+0.590 * x_{5}\right)}{1+\exp \left(\text { intercpt }+0.146 * x_{1}+0.667 * x_{2}+0.375 * x_{3}+0.465 * x_{4}+0.590 * x_{5}\right)}$ $p=\frac{\exp \left(\text { intercept }+0.146 * x_{1}+0.667 * x_{2}+0.375 * x_{3}+0.465 * x_{4}+0.590 * x_{5}\right)}{1+x_{1}}$

b) Decision Tree is a nonparametric method for classification. It uses the Classification and Regression Tree according to the specific variables used (Continues and Categorical) by Classification And Regression Tree (CART) and Chi-squared Automatic Interaction Detection (CHAID)
Table 3 Result of Logistic Regression Analysis for Device Therapy Selection

\begin{tabular}{|c|c|c|c|c|c|}
\hline \multirow[t]{2}{*}{ Variables } & \multirow[t]{2}{*}{$B$} & \multirow{2}{*}{$\begin{array}{l}\mathrm{p}- \\
\text { value }\end{array}$} & \multirow{2}{*}{$\begin{array}{l}\text { Odds } \\
\text { Ratio }\end{array}$} & \multicolumn{2}{|l|}{$95 \% \mathrm{Cl}$} \\
\hline & & & & Lower & $\overline{\text { Upper }}$ \\
\hline \multicolumn{6}{|l|}{ Demographic Variables } \\
\hline Intercept & -23.117 & 0.759 & - & - & - \\
\hline $\mathrm{AHI}$ & -0.005 & 0.931 & 0.995 & 0.773 & 1.121 \\
\hline BMI & 0.146 & $0.045^{*}$ & 1.757 & 1.504 & 3.429 \\
\hline Neck & 0.667 & $0.036^{*}$ & 1.906 & 2.311 & 6.322 \\
\hline Supine Position (S) & 0.004 & 0.807 & 1.004 & 0.933 & 1.094 \\
\hline \multicolumn{6}{|l|}{ PSG Results } \\
\hline REM Duration (min) & 0.375 & $0.031^{*}$ & 1.454 & 1.315 & 3.960 \\
\hline ODI & -0.013 & 0.864 & 0.987 & 0.790 & 1.150 \\
\hline$T<90 \%$ & 0.068 & 0.365 & 1.07 & 0.815 & 1.241 \\
\hline Minimum NREM $\mathrm{SaO}_{2}$ & 0.465 & $0.043^{*}$ & 1.592 & 1.420 & 4.340 \\
\hline Minimum $\mathrm{REM} \mathrm{SaO}{ }_{2}$ & -0.018 & 0.49 & 0.982 & 0.932 & 1.034 \\
\hline Average $\mathrm{SaO}_{2}$ & 0.590 & $0.045^{*}$ & 1.803 & 2.206 & 5.758 \\
\hline \multicolumn{6}{|l|}{ Breath-Holding Maneuver } \\
\hline Breath-Holding Duration & -0.035 & 0.312 & 0.965 & 0.902 & 1.034 \\
\hline Time of $\mathrm{SaO}_{2}$ Recovery & 0.031 & 0.306 & 1.031 & 0.975 & 1.032 \\
\hline $\mathrm{SaO}_{2}$ Post Breath-Holding & 0.451 & 0.372 & 1.569 & 0.583 & 4.229 \\
\hline $\mathrm{SaO}_{2}$ Recovery & 0.149 & 0.565 & 1.16 & 0.789 & 1.929 \\
\hline
\end{tabular}

algorithms. REM Duration less than 12.1 min was considered as the root of the Decision Tree, meaning that REM duration more than 12.1 min showed a $100 \%$ probability of continuing CPAP. In those participants whose REM Duration is less than 12.1 min, minimum $\mathrm{SaO}_{2}$ during NREM was the next level node changing CPAP to BiPAP (Fig. 2). NREM minimum $\mathrm{SaO}_{2}$ levels higher than $79.2 \%$ caused an $87.5 \%$ likelihood of continuing CPAP as the PAP treatment, but levels lower than $84.6 \%$ led to 31.3 and $68.7 \%$ of likelihood for CPAP and BiPAP, respectively (Fig. 2).

Overall analysis of Decision Tree showed an acceptable sensitivity, specificity and positive and negative Likelihood for this model Table 4. Decision Tree analysis shows also a vast area

Table $2 \mathrm{SaO}_{2}$ levels and duration of apnea maneuver in CPAP and BiPAP groups

\begin{tabular}{llll}
\hline $\begin{array}{l}\text { PAP group } \\
\text { Variables }\end{array}$ & CPAP & BiPAP & $\begin{array}{c}p \text { - } \\
\text { value }\end{array}$ \\
\hline Baseline $\mathrm{SaO}_{2}$ & $94.7 \pm 2.1$ & $94.8 \pm 2.4$ & 0.866 \\
Minimum $\mathrm{SaO}_{2}$ & $76.3 \pm 7.8$ & $69.0 \pm 11.9$ & $0.055^{* *}$ \\
Recovery $\mathrm{SaO}_{2}$ & $94.7 \pm 3.5$ & $94.5 \pm 4.7$ & 0.874 \\
Total time of breath-holding maneuver (sec) & $31.9 \pm 17.8$ & $31.0 \pm 11.0$ & 0.852 \\
Time to $\mathrm{SaO}_{2}$ recovery (sec) & $92.8 \pm 29.6$ & $95.0 \pm 33.6$ & 0.226 \\
\hline
\end{tabular}




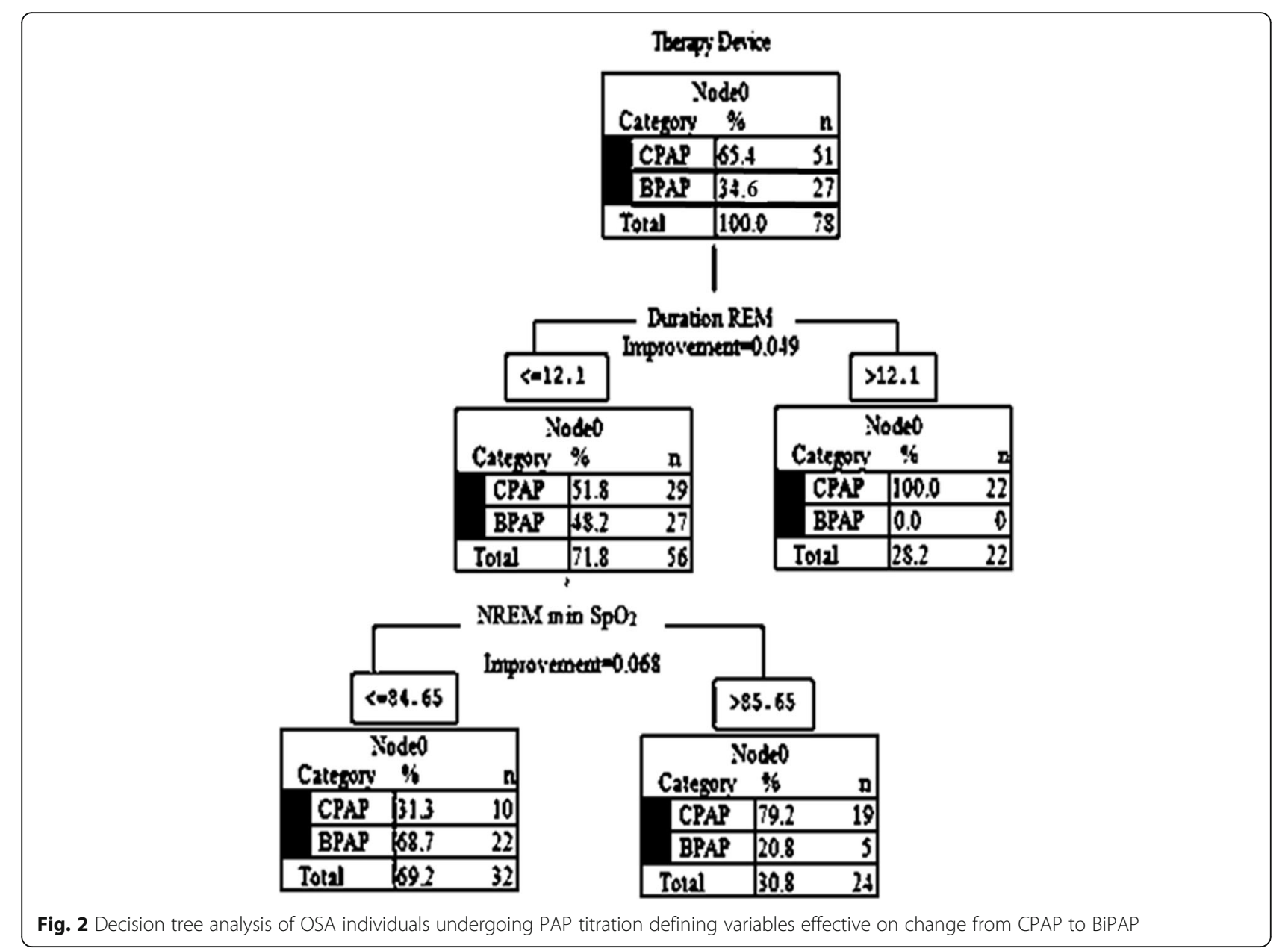

under the curve of ROC (88.6\%) in defining the type of PAP device (Fig. 3).

\section{Discussion}

In developing countries, where patients do not enjoy reimbursement for sleep study, non-invasive mechanical ventilation and CPAP, majority of the patients admitted to the sleep clinics are in moderate to severe OSA stage and cardiovascular disease (Setareh, Mehrnia, \& Mirabi, 2018).In these patient, device titration is recommended in sleep lab. Optimal device titration is a titration that cannot only remove respiratory events while having

Table 4 The Results of ROC analysis

\begin{tabular}{ll}
\hline Statistic & Value \\
\hline Sensitivity & 0.80 \\
Specificity & 0.74 \\
Positive predictive value (PPV) & 0.88 \\
Negative predictive value (NPV) & 0.78 \\
Area under the ROC curve & 0.87 \\
Likelihood ratio + & 2.71 \\
\hline
\end{tabular}

optimum leak but also lets the patient have a comfortable sleep and suitable oxygen saturation. This would be extremely helpful in sleep lab, specifically after midnight when only the technician can attend the patient. On the other hand, in such a situation, changing CPAP into BiPAP can be extremely expensive for the patients, so the more precisely we can decide on the type of device drawing on various parameters, the shorter is the time needed by the technician to make a logical decision. This would ultimately lead to a more reliable night time titration of device.

Two seminal studies in the field of Manual Titration Obstructive Apnea have been introduced according to AASM Guidelines (Berry et al., 2010; Kushida et al., 2008). The first study by Kushida addresses the OSA patients without comorbidities. The study takes into account variables such as the number of respiratory events, supine position, and REM Sleep, but does not provide any protocol for low $\mathrm{SaO}_{2}$ except during events. The second study by Berry is conducted on patients suffering from hypoventilation and OSA, and explains the protocol for utilizing BiPAP in low $\mathrm{SaO}_{2}$ situation during 


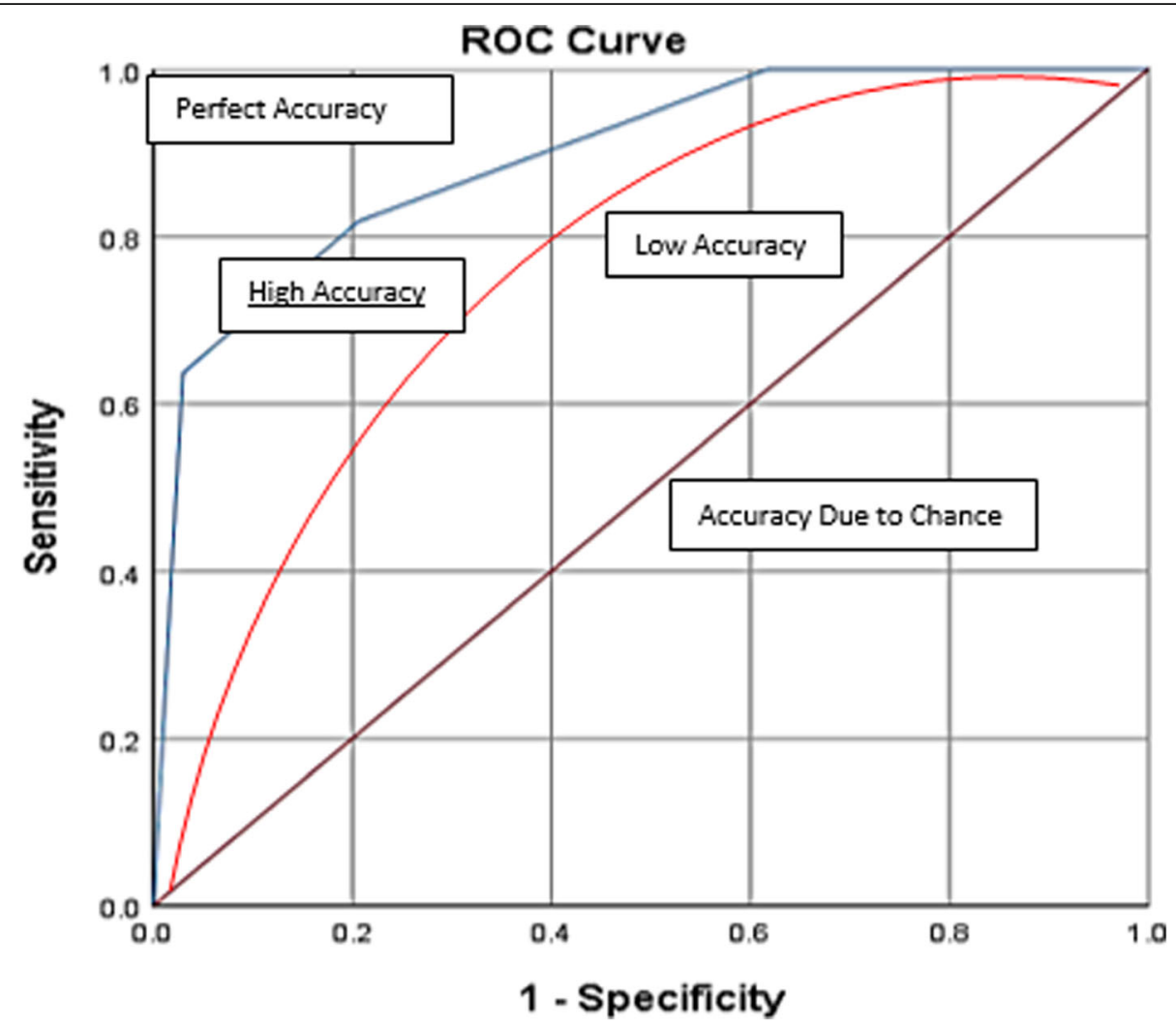

Fig. 3 AUC of ROC for decision tree evaluation (change from CPAP to BiPAP)

wake and sleep from the onset. However, in a number of patients such as those participating in the current study, Baseline $\mathrm{SaO}_{2}$ is $94.7 \%$ which declines to lower than $84.6 \%$ in NREM stage. As a result, this can contribute to the likelihood of changing CPAP to BIPAP (Fig. 2).

Studies on mechanism of cortical control of ventilation showed that the duration of breath-holding can be limited by several factors, such as sensitivity of peripheral chemoreflex, spirometry parameters, the arterial $\mathrm{PCo}_{2}, \mathrm{PO}_{2}$,previous maneuver (Bain et al., 2017; Trembach \& Zabolotskikh, 2018).Ventilatory response to $\mathrm{PCo}_{2}$ and $\mathrm{PO}_{2}$ is considerably variable between patient (West \& Luks, 2016).The duration of voluntary breath-holding doubled after breathing a hyperoxic mixture or after pre-hyperventilation. Those whose minimum post breath-holding $\mathrm{SaO}_{2}$ was lower, might have a higher likelihood of changing CPAP to BiPAP. This might be useful for technicians as a straight forward method to have an estimation of these ventilatory responses and help them to choose BiPAP over CPAP (Table 2). The borderline predictive role for minimums oxygen saturation in voluntary breath-holding maneuver in the study may be limited by variable peripheral chemoreflex and spirometry parameters in these patients in normal range (Bain et al., 2017).
At different levels of hypoxia, there is a nonlinear response to oxygen and most of the responses to level of $\mathrm{PO}_{2}$ are less than $50 \mathrm{mmHg}$. These physiological findings display considerable variability among individuals. Therefore, it seems that measuring oxygen desaturation during NREM sleep and minimum oxygen saturation during voluntary breath-holding maneuver are the predictors of a need of BiPAP during titration with a good trade-off between sensitivity and specificity shown through ROC curve analysis.

Some other studies attempted to find formulas specific for different races. Basoglu et al. proposed a new formula with the same variables among Turkish population (Basoglu \& Tasbakan, 2012).Their formula utilized neck circumference (NC) and oxygen desaturation index (ODI). It can be easily concluded from every titration protocol that more severe OSA patients need BiPAP more frequently (Kushida et al., 2008). Our results were in concordance with more severe OSA and lower $\mathrm{SaO}_{2}$ during NREM sleep.

Camacho et al. in their systematic review of 26 studies on mathematical equations of CPAP prediction, reported BMI and mean oxygen saturation as the most heavily weighted variables along with BMI, AHI, and neck circumference as the most frequently used variables (Camacho et al., 2015). 
The likelihood of changing from CPAP to BiPAP was almost doubled by higher BMI and neck circumference in our study population. Meanwhile, Hoffstein formula (Miljeteig \& Hoffstein, 1993) is the most widely used CPAP prediction tool worldwide, which uses BMI, AHI, and neck circumference. Although it is widely used for many years and validated in many different studies, this study suggests that these parameters were not enough compared to other factors (Fig. 2).

OSA is also a well-known etiology of REM deprivation. OSA causes the reduction in REM sleep percentage. The more intense OSA, the shorter the length of REM (Kimoff, 1996; Wang et al., 2015).Therefore, a relative short REM can indicate an increase in the probability of need for BiPAP.

The present study was designed to evaluate BiPAP versus CPAP preference by anthropometric, polysomnographic, and other type of data on a clinical maneuver called voluntary breath-holding maneuver. We used decision tree analysis to find if there is any relationship between patient's variables and the need to use BiPAP or CPAP, by using some of the most common polysomnographic and anthropometric variables. Although these results cannot render the titration procedure useless completely, it is one of the limitations of the study but can help the technicians to pay attention to special variables in order to save time, specifically during splitnight titration and to choose the more suitable device as soon as possible.

The main focus in this study was using of logistic regression analysis as a parametric model to find an equation between independent variables with respect to its dependent variable. Technically by logistic regression application, it was estimated the effect of each independent variable on the odds of change of device therapy from BIPAP to CPAP.On the other side, we gained advantages of the decision tree to find out considerable overlap between the variables used in the logistic regression equation and the decision tree. It might be suspected that the two procedures are picking the same cases like what was seen about REM duration and NREM minimum $\mathrm{SaO}_{2}$.

\section{Conclusion}

Minimum oxygen following voluntary breath-holding maneuver along with higher BMI and larger neck circumference are predictors of the need to use BiPAP. Shorter REM duration was another predictor of higher chance of BiPAP during titration of the present OSA participants.

Future research projects can have more homogeneous forced vital capacity (FVC), forced expiratory volume in $1 \mathrm{~s}\left(\mathrm{FEV}_{1}\right)$, and investigate and explore a host of other unknown factors in order to avoid having a heterogeneous sample, which seems to be the main cause of obtaining a borderline $P$ value regarding minimum $\mathrm{SaO}_{2}$ in breathholding maneuver.

\begin{abstract}
Abbreviations
AASM: American Academy of Sleep Medicine; AHI: Apnea Hypopnea Index; BiPAP: Bilevel Positive Airway Pressure; BMI: Body Mass Index; CAD: Coronary Artery Disease; CART: Covering both classification and regression; CHAID: Chi-squared automatic interaction Detection; CPAP: Continuous Positive Airway Pressure; DM: Diabetes Mellitus; EPAP: Expiratory positive airway pressure; $\mathrm{FEV}_{1}$ : Forced Expiratory Volume in $1 \mathrm{~s}$; FVC: Forced Vital Capacity; HTN: Hypertension; IPAP : Inspiratory positive airway pressure; NC: Neck Circumference; NREM Sleep: Non REM Sleep; ODI: Oxygen Desaturation Index; OSA: Obstructive Sleep Apnea; PAP: Positive Airway Pressure; PSG: Polysomnography; REM Sleep: Rapid Eye Movement Sleep; ROC: Receiver Operating Characteristic; SaO2: Oxygen Saturation; TRT: Total Recording Time; TST: Total Sleep Time
\end{abstract}

\section{Acknowledgements}

Authors kindly appreciate Aram Karami Rad and Naeime Mohammadi as the technicians of sleep laboratories for their great cooperation in the study.

\section{Funding}

This study is supported by research grant form deputy of research of National Research Institute of Tuberculosis and Lung Diseases

\section{Availability of data and materials}

The datasets used and/or analyzed during the current study are available from the corresponding author on reasonable request.

\section{Authors' contributions}

JS designed the study, helped in data collection and final edition of the article, MM helped in data collection and final edition of the article, MA wrote a part of article draft, BK analyzed data and wrote the result of the study and PAN designed and supervised the study and final edition of the article. All authors read and approved the final manuscript.

Ethics approval and consent to participate

Informed consent was obtained from all individual participants included in the study. The current study is approved by ethics committee of NRITLD number of IR.SBMU.NRITLD.1397.530.

Consent for publication

Not applicable.

\section{Competing interests}

The authors declare that they have no competing interests.

\section{Publisher's Note}

Springer Nature remains neutral with regard to jurisdictional claims in published maps and institutional affiliations.

\footnotetext{
Author details

${ }^{1}$ Psychiatry and Behavioral Sciences Research Center, Addiction Institute, Mazandaran University of Medical Sciences, Sari, IR, Iran. ${ }^{2}$ Tracheal Diseases Research Center, National Research Institute of Tuberculosis and Lung Diseases (NRITLD), Shahid Beheshti University of Medical Sciences, Tehran, Iran. ${ }^{3}$ Lung Diseases Research Center, Mashhad University of Medical Sciences, Faculty of Medicine, Mashhad, Iran. ${ }^{4}$ Clinical Tuberculosis and Epidemiologic Research Center, National Research Institute of Tuberculosis and Lung Diseases (NRITLD), Shahid Beheshti University of Medical Sciences, Tehran, Iran. ${ }^{5}$ Masih Daneshvari Hospital, PO Box: 19575-154, Daar-Abad, Niavaran, Tehran Zip Code: 19569-44413, Iran.
} 
Received: 4 October 2018 Accepted: 7 February 2019

Published online: 15 February 2019

\section{References}

Abdel Wahab N, Ahmed YN. Optimal level of continuous positive airway pressure: auto-CPAP titration versus predictive formulas. Egyptian J Chest Dis Tuberc. 2017;66(2):353-61. https://doi.org/10.1016/j.ejcdt.2016.11.004.

Antonescu-Turcu A, Parthasarathy S. CPAP and bi-level PAP therapy: new and established roles. Respir Care. 2010;55(9):1216-29.

Bain, A. R., Barak, O. F., Hoiland, R. L., Drvis, I., Bailey, D. M., Dujic, Z., .. Ainslie, P. N. (2017). Forced vital capacity and not central chemoreflex predicts maximal hyperoxic breath-hold duration in elite apneists. Respir Physiol Neurobiol, 242, 8-11. doi: https://doi.org/10.1016/j.resp.2017.02.015.

Basoglu OK, Tasbakan MS. Determination of new prediction formula for nasal continuous positive airway pressure in Turkish patients with obstructive sleep apnea syndrome. Sleep Breath. 2012;16(4):1121-7. https://doi.org/10.1007/ s11325-011-0612-z.

Berry, Richard B., Chediak, Alejandro, Brown, Lee K., Finder, Jonathan, Gozal, David, Iber, Conrad, ... Medicine, Nppv titration task force of the American Academy of sleep. (2010). Best clinical practices for the sleep center adjustment of noninvasive positive pressure ventilation (NPPV) in stable chronic alveolar hypoventilation syndromes J. Clin. Sleep Med. 6(5), 491-509.

Camacho M, Riaz M, Tahoori A, Certal V, Kushida CA. Mathematical equations to predict positive airway pressures for obstructive sleep apnea: a systematic review. Sleep Disord. 2015;2015:293868. https://doi.org/10.1155/2015/293868.

Camacho, M., Ruoff, C. M. Kawai, M., Modi, R. Arbee, J. Hekmat, A. ... Kushida, C. A. (2016). Five-minute awake snoring test for determining CPAP pressures (five-minute CPAP test): a pilot study. Sleep Disord, 2016, 7380874. doi: https://doi.org/10.1155/2016/7380874.

Carlucci, Annalisa, Ceriana, Piero, Mancini, Marco, Cirio, Serena, Pierucci, Paola, D'Artavilla Lupo, Nadia, ... Fanfulla, Francesco. (2015). Efficacy of Bilevel-auto Treatment in Patients with Obstructive Sleep Apnea Not Responsive to or Intolerant of Continuous Positive Airway Pressure Ventilation J. Clin. Sleep Med., 11(9), 981-985. doi: https://doi.org/10.5664/jcsm.5008.

Chow SC, Shao J, WangH. Sample Size calculation in clinical Research, 2nd ed. New York: Chapman and Hall/CRC Press, Taylor \& Francis, 16; 2003.

Ebben MR, Narizhnaya M, Krieger AC. A new predictive model for continuous positive airway pressure in the treatment of obstructive sleep apnea. Sleep Breath. 2017;21(2):435-42. https://doi.org/10.1007/s11325-016-1436-7.

Kimoff RJ. Sleep fragmentation in obstructive sleep apnea. Sleep. 1996;19(9 Suppl):S61-6.

Kushida, C. A., Chediak, A., Berry, R. B., Brown, L. K., Gozal, D., Iber, C., .. Rowley, J. A. (2008). Clinical guidelines for the manual titration of positive airway pressure in patients with obstructive sleep apnea. J Clin Sleep Med, 4(2), 157-171.

Miljeteig $\mathrm{H}$, Hoffstein V. Determinants of continuous positive airway pressure level for treatment of obstructive sleep apnea. Am Rev Respir Dis. 1993;147(6 Pt 1):1526-30. https://doi.org/10.1164/ajrccm/147.6_Pt_1.1526.

Noseda A, Andre S, Potmans V, Kentos M, de Maertelaer V, Hoffmann G. CPAP with algorithm-based versus titrated pressure: a randomized study. Sleep Med. 2009;10(9):988-92. https://doi.org/10.1016/.sleep.2008.08.010.

Setareh J, Mehrnia M, Mirabi A. The risk of obstructive sleep apnea and daytime Sleepinessin patients with cardiovascular disease. J Mazandaran Univ Med Sci. 2018:28(167):29-41.

Trembach NV, Zabolotskikh IB. Voluntary breath-holding duration in healthy subjects with obesity: role of peripheral chemosensitivity to carbon dioxide. Respir Physiol Neurobiol. 2018;249:7-10. https://doi.org/10.1016/j.resp.2017.12.006.

Wang YQ, Li R, Zhang MQ, Zhang Z, Qu WM, Huang ZL. The neurobiological mechanisms and treatments of REM sleep disturbances in depression. Curr Neuropharmacol. 2015:13(4):543-53.

West JB, Luks A. West's respiratory physiology : the essentials; 2016.

Ready to submit your research? Choose BMC and benefit from:
- fast, convenient online submission
- thorough peer review by experienced researchers in your field
- rapid publication on acceptance
- support for research data, including large and complex data types
- gold Open Access which fosters wider collaboration and increased citations
- maximum visibility for your research: over 100M website views per year
At BMC, research is always in progress.
Learn more biomedcentral.com/submissions

